\title{
Molecular cloning of the mouse homologue of Rab3c
}

\section{N J Pavlos, J Xu, J M Papadimitriou ${ }^{1}$ and M H Zheng}

Department of Surgery (Orthopaedics), University of Western Australia, QEII Medical Centre, Perth, Western Australia, 6009

${ }^{1}$ Department of Pathology, University of Western Australia, QEII Medical Centre, Perth, Western Australia, 6009

(Requests for offprints should be addressed to M H Zheng, Department of Surgery (Orthopaedics), University of Western Australia, 2nd Floor M Block, QEII Medical Centre, Nedlands, Western

Australia 6009; Email: zheng@cyllene.uwa.edu.au)

\begin{abstract}
Small GTP-binding proteins of the Rab subfamily are key regulators of intracellular vesicle transport. Here we report the isolation of a cDNA clone encoding the complete Rab3c isoform from mouse embryo using a degenerative PCR-based approach. Multiple sequence alignment revealed that the predicted amino acid sequence was identical to the previously identified rat Rab3c isoform and 98\% identical to the published bovine Rab3c GTPase from brain. Furthermore by in situ hybridisation, Rab3c mRNA was detectable within various regions
\end{abstract}

of the brain, cartilage and highly enriched within intestinal villi of foetal tissues. Chondrocytes in the hypertrophic zone, but not reserve or proliferative zones, expressed high levels of Rab3c. This pattern of expression corresponds with the genesis of matrix vesicles during endochondral ossification. In all, our results suggest that in addition to its functional role during regulated secretion in brain, Rab3c may play a part in matrix vesicle trafficking during skeletal development.

Fournal of Molecular Endocrinology (2001) 27, 117-122

\section{INTRODUCTION}

In eukaryotic cells, inter-organellar transport of proteins is mediated by small carrier vesicles, which bud from a donor compartment, migrate through the cytoplasm and identify and fuse with target membrane(s). Rab proteins, a family of small (21-25 kDa) Ras-like GTP-binding proteins, have long been recognised as key regulators of membrane trafficking events and are thought to confer the specificity of vesicle docking during endocytosis, transcytosis and regulated secretion (Novick \& Zerial 1997, Zerial \& Stenmark 1993). Over 60 Rabs have been identified to date, each displaying differential cellular and subcellular localisation patterns suggesting that individual members of the Rab superfamily may play discrete roles in certain cell types (Stenmark \& Olkkonen 2001).

One of the most intensely studied subfamilies of the Rab GTPases are the Rab3 isoforms which consist of four members, Rab3a, -b, -c and -d (Matsui et al. 1988, Touchot et al. 1987, Zahraoui et al. 1989). Although Rab3 proteins are highly homologous, displaying $77-85 \%$ amino acid identity with greatest variance evident within the $\mathrm{N}$ - and
C-terminal regions, their subcellular targets and functional roles remain somewhat distinct between them (Baldini et al. 1992, Fischer von Mollard et al. 1990, Fischer von Mollard et al. 1994). Rab3a and Rab3b isoforms are preferentially expressed in neurons and neuroendocrine cells where they have been localised to synaptic vesicles (Fischer von Mollard et al. 1990) and chromaffin granules (Darchen et. al. 1995), whereas Rab3d exhibits primary expression in non-neuron cells including mast cells, gastric chief cells and adipocytes (Baldini et al. 1992, Raffaniello et al. 1996, Roa et al. 1997). At present however, little information is available concerning the expression and functional role of Rab3c, although it has been shown to co-enrich with Rab3a on synaptic vesicles (Fischer von Mollard et al. 1994) and, like its other Rab3 counterparts, is thought to guide membrane fusion between transport vesicles and their target membranes during regulated exocytosis (Fischer von Mollard et al. 1994, Geppert et al. 1997). Here we have isolated a cDNA clone encoding the mouse homologue of the Rab3c protein from mouse embryo using a degenerative oligonucleotide PCRbased approach. In addition by in situ hybridisation, 
we detected the expression of Rab3c mRNA transcripts in mouse embryos at various stages of skeletal development and embryogenesis.

\section{MATERIALS AND METHODS}

A 15 day old mouse embryo was used as a template for cloning of Rab3 proteins. Rab3 degenerative oligonucleotide primers were designed based on the conserved sequences of Rab3 and Ras consensus amino acid sequences (QNFDYM) and (WDNAQV). Total RNA was extracted from 15 day old mouse embryo (C57 black) with RNAzol B (Tel Test). PCR was performed using DyNAzyme polymerase (FINNZYMES) with sense and antisense primers: (+) 5'-CARAAYTTYGAYTAYA TG-3' and (-) 5'-NACYTGNGCRTTRTCCCA3'. A $342 \mathrm{bp}$ amplimer corresponding to the predicted size was gel purified using the UltraClean GelSpin Purification Kit (MO BIO Laboratories Inc., CA, USA) and subcloned into a pCR2.1 TA Cloning Vector (Invitrogen, CA, USA). Colony lift hybridisation and restriction digest analyses were utilised for positive clone selection. Putative clones were sequenced using an automated ABI 373 system and, of these, a clone, displaying striking homology with rat and bovine Rab3c was identified. $3^{\prime}$ and $5^{\prime}$ end rapid amplification of cDNA ends (RACE) strategies were employed using a modification of the published protocol from Frohman et al. (Frohman et al. 1988) to obtain the full length of the mouse Rab3c coding sequence. Blast $\mathrm{N}$ (Altschul et al. 1990) at the NCBI was used to screen for DNA and protein homologies via the World Wide Web.

For in situ hybridisation, PCR was used to generate a cDNA product that partially encoded the Rab3c C- terminal region. Oligonucleotide primers were directed against amino acid sequences that were specific to the Rab3c GTPase; Forward: Rab3c1 5'-GAGAGCTTGGAAACCGAC-3' Re-

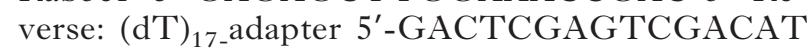
CGATTTTTTTTTTTTTTTTT-3'. Amplimers were gel purified and cloned into the $\mathrm{pCR} 2 \cdot 1$ vector using the Original TA Cloning Kit (Invitrogen, CA, USA). Recombinant plasmids containing
Rab3c inserts were linearized and transcribed into Digoxingenin (DIG)- labelled anti-sense riboprobes with T7 RNA polymerase, using a DIG RNA labelling Kit (Boehringer Mannheim, Germany). All clones were sequenced for the confirmation of orientation and authenticity of genes. in situ hybridisation was performed on $7,11,15$ and 19-day-old whole foetal mouse paraffin embedded sections under high stringency conditions as previously described (Huang et al. 2000). Colour development was carried out with NBT/BCIP stock solution (Boehringer Mannheim, Germany). Signals were assessed by light microscopy (Leitz).

\section{RESULTS AND DISCUSSION}

A full-length $1.0 \mathrm{~kb}$ clone of the mouse Rab3c isoform was identified. The mouse Rab3c cDNA, GenBank accession number AY026947, contained an open reading frame (ORF) of $684 \mathrm{bp}$, encoding a putative polypeptide of 227 amino acid residues (Fig. 1). In addition, the $1.0 \mathrm{~kb}$ clone encompassed $5^{\prime}(69 \mathrm{bp})$ and $3^{\prime}$ (254 bp) untranslated sequences that contained a potential polyadenylation signal (TATAAA) $23 \mathrm{bp}$ upstream of the $3^{\prime}$ poly $\mathrm{A}^{+}$ region. The ORF of this sequence matched a mouse Rab3c that was recently deposited with Genbank (T C Sudhof, unpublished data; Accession No: AF312037). The mouse Rab3c isoform showed characteristic structural motifs of Rab proteins including five conserved GTP-binding domains (G1-G5), an effector binding domain (within G-2) which associates with GTPase activating proteins and a carboxy-terminal cysteine-motif (CGC) which has been shown to be obligatory for isoprenylation and membrane attachment (Bourne et al. 1991, Chavrier et al. 1991).

Multiple sequence alignment of the mouse Rab3c protein with previously identified mammalian Rab3 isoforms revealed a high degree of cross-species conservation. The amino acid sequence of mouse Rab3c is strikingly identical to that of the published rat Rab3c isoform from PC12 cells (Viggeswarapu \& Wildey 1996) and $98 \%$ identical to the previously characterised bovine sequence from brain

\footnotetext{
FIGURE 1. (A) Nucleotide and deduced amino acid sequence of the mouse Rab3c cDNA spanned by its $5^{\prime}$ and $3^{\prime}$ untranslated regions. The open reading frame is at 70-753 given in upper case. Amino acid sequence is represented in bold. Stop codon is marked by an asterisk (GenBank Accession number AY026947). Boxed region defines the location of the cDNA probe used for in situ experiments. (B) Alignment of the predicted amino acid sequences of mouse Rab3C with rab3 sequences from various mammalian species (GenBank Acession: D78197; M19887; X72966; AF312036, M89777). The conserved structural motifs are highlighted: G-1 - G-5 domains, switch I, and switch II regions. The putative effector-binding domain is indicated by (ED) and site of geranylgeranylation (CXC) is marked. Identical amino acid residues are boxed in black, conservative changes are given in grey.
} 
A

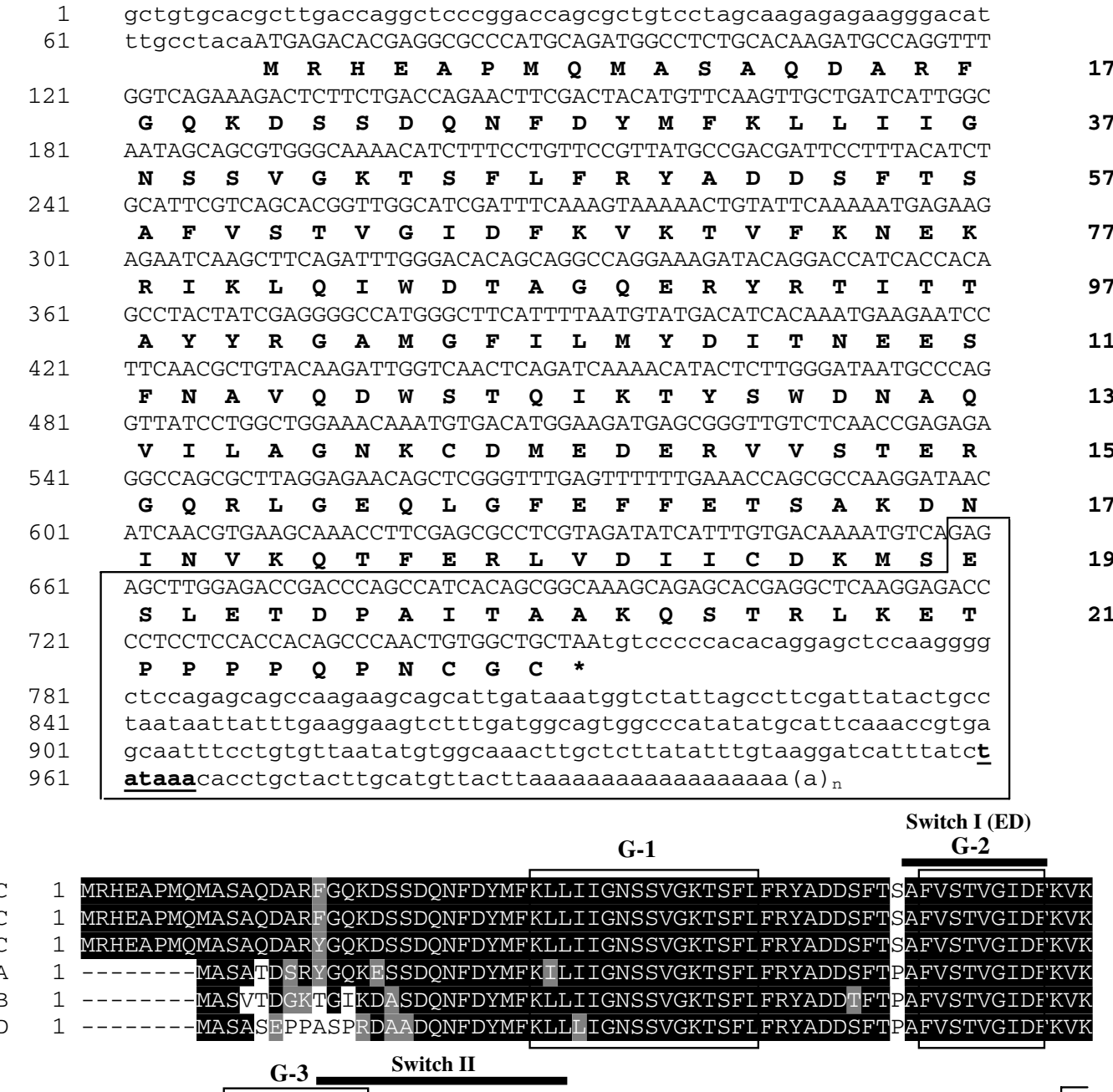

Rat

Rab3C

Rab3c

Bovine Rab3C

Mouse Rab3A

Mouse Rab3B

Mouse Rab3D

TVFKNEKRIKLQIWDTAGQERYRTITTAYYRGAMGF ILMYDITNEESFNAVQDWSTQIKTYSWDNAQVII TVYRHEKRVKLOIWDTAGQFRYRTITTAYYRGAMGF ILMYDITNEESFNAVODWATOI KTYSWDNAQVII

Rat G-4

\section{G-4}

G-5

Mouse Rab3C 141 Bovine Rab3C 140 Mouse Rab3A 133 Mouse Rab3B 133 Mouse Rab3D 133

Rat Rab3C 210 Mouse Rab3C 210 Bovine Rab3C 209 Mouse Rab3A 203 Mouse Rab3B 202 Mouse Rab3D 202
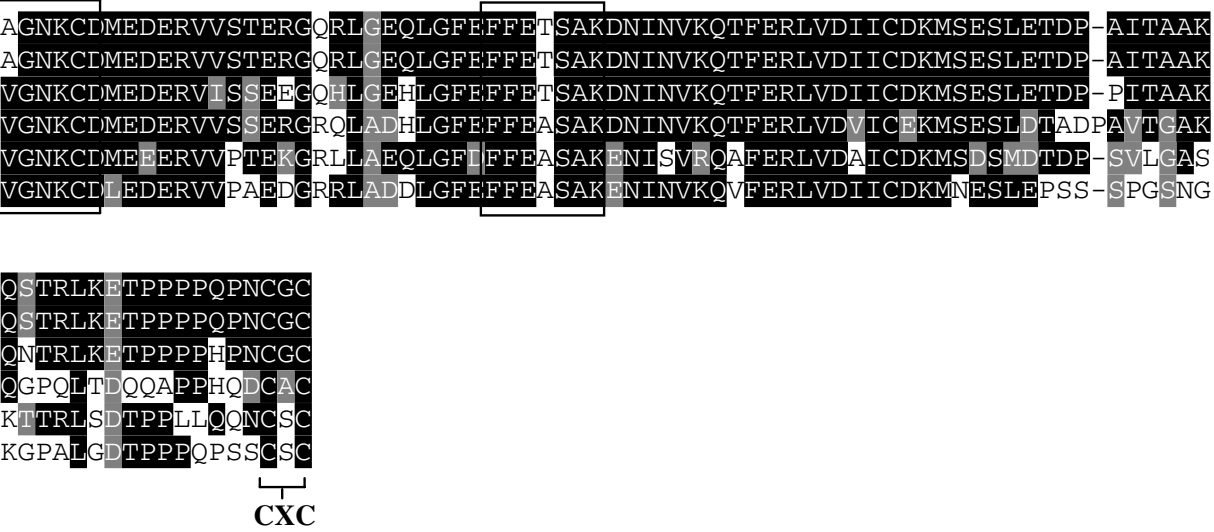


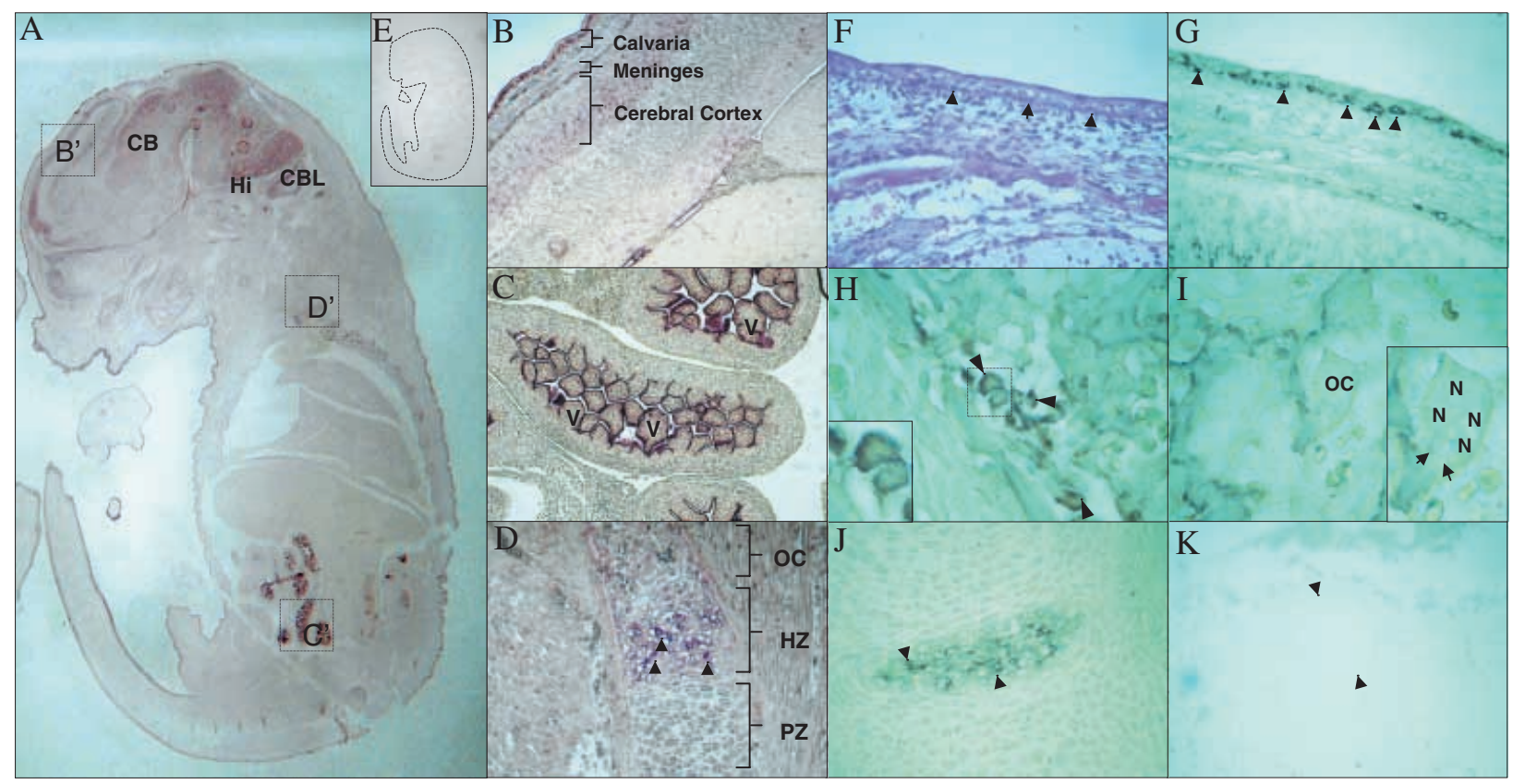

FIGURE 2. In situ hybridisation of Rab3c in developing mouse embryos. Paraffin embedded sections of 15 and 19 day old foetal mice were hybridised with an anti-sense riboprobe for Rab3c and assessed by bright field microscopy following colour development. (A) Low magnification overview of a foetal mouse (day 15) showing strong staining for Rab3c mRNA within various regions of the brain (B), intestine $(\mathrm{C})$ and developing skeleton (D). Abbreviations: CB: cerebrum, CBL: cerebellum, Hi: hippocampus. in situ hybridisation following RNAse treatment revealed only background activity and served as a control, $\times 10(\mathrm{E})$. (B) High magnification of Rab3c signal within the mouse brain. A strong signal can be observed within the cerebral cortex and connective tissue meninges. Staining is also apparent in bone lining cells within the calvaria, $\times 100$. (C) A magnified view of the foetal mouse intestine shows Rab3c mRNA signal enriched within epithelial cells of the intestinal villi $=\mathrm{V}, \times 200$. (D) Enlargement of a calcifying rib, revealing strong staining within chondrocytes (arrowheads) of the hypertrophic zone: $\mathrm{HZ}$. PZ and OC denote proliferative zone and ossifying cartilage. Rab3c mRNA within skeletal elements of a 19 day old mouse embryo (F-K). (F) H\&E section of the developing calvaria. (G-H) High magnification of foetal calvaria, arrowheads indicate positive bone-lining cells, $\times$ 400. (I) A resident bone resorbing osteoclast $(\mathrm{OC})$, arrows define sealing zone, $\mathrm{N}=$ nucleus, $\times 400$. (J) Section of a mineralising vertebra shows Rab3c mRNA expression in hypertrophic chondrocytes. (K) RNAse treated control, arrowheads define an island of hyaline cartilage within a vertebra.

(Matsui et al. 1988). In addition, the predicted mouse Rab3c isoform shares up to $85 \%, 80 \%$ and $77 \%$ amino acid identity with its mouse Rab3a, -b, and -d isotypes respectively. Highest interspecies and subfamily conservation was observed within the regions that are directly involved in guanine nucleotide binding and hydrolysis (effector/switch I and switch II) (Bourne et al. 1991). Moreover the greatest protein divergence was observed within the Rab3c C-terminal region that is thought to bestow the specificity of the proteins to their intracellular compartments (Chavrier et al. 1991).

To examine the expression of Rab3c genetranscripts at the tissue and cellular level in developing mouse embryos we performed nonradioactive mRNA in situ hybridisation on whole foetal mouse sections (days 7, 11, 15 and 19 of gestation) using digoxigenin-labelled anti-sense riboprobes, specific for the $\mathrm{C}$-terminus of rab3C. No obvious Rab3c expression was detected in any tissue at days 7 and 11 (data not shown). In contrast, Rab3c mRNA expression was observed within various tissues upon days 15 (Fig. 2, panels A-E) and 19 of foetal development (Fig. 2, panels F-K).

As shown in Figure 2A, a strong staining pattern for Rab3c mRNA was observed throughout the foetal mouse brain. More specifically, strong signal was observed in the cerebral cortex, outer layers of the cerebellum with moderate staining evident within the hippocampus (panels A and B). These results were consistent with previous tissue expression studies of rat and bovine Rab3c by Northern and Western blot analyses (Su et al. 1994, 
Viggeswarapu \& Wildey 1996). RNAse treatment prior to hybridisation displayed little colour development in foetal sections indicating the specificity of the probes for target mRNA sequence (panels $\mathrm{E}$ and $\mathrm{K}$ ).

In addition to brain, Rab3c mRNA transcripts were also localised to a number of non-neuronal tissue and cell types. A strong, well-localised staining pattern was observable in the foetal intestine (panel C). Upon closer examination, the reaction product was clearly evident within the epithelium of the intestinal villi. This expression pattern is analogous with previously identified Rab18 and Rab20 GTPases, both of which have been localised to epithelial cells of mouse foetal intestine (day 16.5) and are thought to mediate apical and basolateral endocytic trafficking in polarised cells (Lutcke et al. 1994). Whether Rab3c plays a similar, or functionally distinct role in polarised cells remains to be clarified; however, this study indicates for the first time a role for Rab3c in polarised vesicular transport.

Rab3c gene transcripts were also detectable within bone-lining cells (osteoblastic lineage cells) of both 15 (panel B) and 19 (panels F-H) day foetal calvaria; however, no mRNA signal was apparent within residing multinucleated osteoclasts (panel I). Moderate staining was also discernible within calcifying vertebrae (panel J). More interestingly however, was the observed staining pattern of Rab3c within areas of endochondral ossification as shown in panels $\mathrm{D}$ and $\mathrm{J}$. Rab3c mRNA was observed in chondrocytes of hyaline cartilage, in particular, within the hypertrophic layer but not in the rest or proliferating zones. Thus the pattern of Rab3c expression appears to specifically correlate with the production of chondrocytic matrix vesicles. In contrast, Rab3d mRNA expression was detected in osteoclasts, but not mesenchymal lineage cells, by Northern Blot analysis in situ hybridisation (data not shown).

Endochondral ossification, a complex multi-step process, is essential for skeletal development during embryogenesis. It is now well accepted that deposition of matrix vesicles at the extracellular compartment of chondrocytes initiates the process of mineralization (Anderson 1995). During endochondral ossification chondrocytes are induced from a resting stage to a proliferating stage and then characteristically undergo hypertrophy. One of the distinguishing features of enlarging chondrocytes within the hypertrophic zone is the production of matrix vesicles (Anderson 1995). Matrix vesicles, small extracellular membranous particles containing alkaline phosphatase and ATPase (Boyan et al. 1989, Matsuzawa \& Anderson 1971), have been shown to dock at the side of polarised cytoplasmic membranes of chondrocytes (Glaser \& Conrad 1981, Suzuki et al. 1981) and osteoblasts (Boyan et al. 1989, Sudo et al. 1983) where they then bud from the membrane and are subsequently expelled into the extracellular matrix. The restriction of matrix vesicle deposition defines the spatial regulation of the process. Given that the expression pattern of Rab3c in hypertrophic chondrocytes is comparable to matrix vesicle genesis, it is tempting to speculate that Rab3c plays a role in the deposition of matrix vesicles during mineralization. To this end, whether or not Rab3c mediates the docking, budding and/or release of matrix vesicles by hypertrophic chondrocytes requires further study.

In summary, we have cloned a complete cDNA encoding the mouse Rab3c GTP-binding protein by a degenerative-PCR strategy. The predicted amino acid sequence was identical to the previously identified rat Rab3c isoform from PC12 cells and 98\% identical to the published bovine Rab3c GTPase isolated from brain. Using whole mouse in situ hybridisation, we have shown that Rab3c was expressed not only in brain tissue but also hypertrophic hyaline cartilage, bone-lining cells and intestinal villi. Our results suggest that Rab3c may play a role in vesicle transport in non-neuron cells, in particular the hypertrophic chondrocytes.

\section{ACKNOWLEDGMENTS}

This work was supported by grants from the National Health and Medical Research Council of Australia.

This work was presented at the 10th Annual Scientific Meeting of Australia and New Zealand Bone and Mineral Society in November 2000.

\section{REFERENCES}

Altschul SF, Gish W, Miller W, Myers EW \& Lipman DJ 1990 Basic local alignment search tool. Fournal of Molecular Bioiogy 215 403-410.

Anderson HC 1995 Molecular biology of matrix vesicles. Clinical Orthopedics 314 266-280.

Baldini G, Hohl T, Lin HY \& Lodish HF 1992 Cloning of a Rab3 isotype predominantly expressed in adipocytes. PNAS 89 5049-5052.

Bourne HR, Sanders DA \& McCormick F 1991 The GTPase superfamily: conserved structure and molecular mechanism. Nature 349 117-127.

Boyan BD, Schwartz Z, Bonewald LF \& Swain LD 1989 Localization of 1,25-(OH)2D3-responsive alkaline phosphatase in osteoblast-like cells (ROS17/2.8, MG 63, and MC 3T3) and growth cartilage cells in culture. Fournal of Biological Chemistry 264 11879-11886.

Chavrier P, Gorvel JP, Stelzer E, Simons K, Gruenberg J \& Zerial M 1991 Hypervariable C-terminal domain of rab proteins acts as a targeting signal. Nature 353 769-772. 
Darchen F, Senyshyn J, Brondyk WH, Taatjes D J, Holz RW, Henry JP, Denizot JP \& Macara G 1995 The GTPase Rab3a is associated with large dense core vesicles in bovine chromaffin cells and rat PC12 cells. Fournal of Cell Science 108 1639-1649.

Fischer von Mollard G, Mignery GA, Baumert M, Perin MS, Hanson TJ, Burger PM, Jahn R \& Sudhof TC 1990 rab3 is a small GTP-binding protein exclusively localized to synaptic vesicles. PNAS 87 1988-1992.

Fischer von Mollard G, Stahl B, Khokhlatchev A, Sudhof TC \& Jahn R 1994 Rab3C is a synaptic vesicle protein that dissociates from synaptic vesicles after stimulation of exocytosis. Fournal of Biological Chemistry 269 10971-10974.

Frohman MA, Dush MK \& Martinm GR 1988 Rapid production of full-length cDNAs from rare transcripts: amplification using a single gene-specific oligonucleotide primer. PNAS 85 8998-9002.

Geppert M, Goda Y, Stevens CF \& Sudhof TC 1997 The small GTP-binding protein Rab3A regulates a late step in synaptic vesicle fusion. Nature 387 810-814.

Glaser JH \& Conrad HE 1981 Formation of matrix vesicles by cultured chick embryo chondrocytes. Fournal of Biological Chemistry 256 12607-12611.

Huang L, Xu J, Wood DJ \& Zheng MH 2000 Gene expression of osteoprotegerin ligand, osteoprotegerin, and receptor activator of NF-kappaB in giant cell tumor of bone: possible involvement in tumor cell-induced osteoclast-like cell formation. American Fournal of Pathology 156 761-767.

Lutcke A, Parton RG, Murphy C, Olkkonen VM, Dupree P, Valencia A, Simons K \& Zerial M 1994 Cloning and subcellular localization of novel rab proteins reveals polarized and cell type-specific expression. Fournal of Cell Science 107 3437-3448.

Matsui Y, Kikuchi A, Kondo J, Hishida T, Teranishi Y \& Takai Y 1988 Nucleotide and deduced amino acid sequences of a GTP-binding protein family with molecular weights of 25000 from bovine brain. Fournal of Biological Chemistry 263 11071-11074.

Matsuzawa T \& Anderson HC 1971 Phosphatases of epiphyseal cartilage studied by electron microscopic cytochemical methods. Fournal of Histochemistry and Cytochemistry 19 801-808.
Novick P \& Zerial M 1997 The diversity of Rab proteins in vesicle transport. Current Opinions in Cell Biology 9 496-504.

Raffaniello RD, Lin J, Wang F \& Raufman JP 1996 Expression of Rab3D in dispersed chief cells from guinea pig stomach. Biochimica Biophysica Acta 1311 111-116.

Roa M, Paumet F, Le Mao J, David B \& Blank U 1997 Involvement of the ras-like GTPase rab3d in RBL-2H3 mast cell exocytosis following stimulation via high affinity $\mathrm{IgE}$ receptors (Fc epsilonRI). Fournal of Immunology 15 2815-2823.

Stenmark H \& Olkkonen VM 2001 The Rab GTPase family. Genome Biology 23007.

Su YC, Kao LS, Chu YY, Liang Y, Tsai MH \& Chern Y 1994 Distribution and regulation of rab3C, a small molecular weight GTP-binding protein. Biochemical and Biophysical Research Communications 200 1257-1263.

Sudo H, Kodama HA, Amagai Y, Yamamoto S \& Kasai S 1983 in vitro differentiation and calcification in a new clonal osteogenic cell line derived from newborn mouse calvaria. Fournal of Cell Biology 96 191-198.

Suzuki F, Takase T, Takigawa M, Uchida A \& Shimomura Y 1981 Simulation of the initial stage of endochondral ossification: in vitro sequential culture of growth cartilage cells and bone marrow cells. PNAS 78 2368-2372.

Tang LH, Gumkowski FD, Sengupta D, Modlin IM \& Jamieson JD 1996 rab3D protein is a specific marker for zymogen granules in gastric chief cells of rats and rabbits. Gastroenterology 110 809-820.

Touchot N, Chardin P \& Tavitian A 1987 Four additional members of the ras gene superfamily isolated by an oligonucleotide strategy: molecular cloning of YPT-related cDNAs from a rat brain library. PNAS 84 8210-8214.

Viggeswarapu M \& Wildey GM 1996 Cloning and tissue expression of the rat RAB 3C GTP-binding protein. Biochemical and Biophysical Research Communications 227 645-650.

Zahraoui A, Touchot N, Chardin P \& Tavitian A 1989 The human Rab genes encode a family of GTP-binding proteins related to yeast YPT1 and SEC4 products involved in secretion. Fournal of Biological Chemistry 264 12394-12401.

Zerial M \& Stenmark H 1993 Rab GTPases in vesicular transport. Current Opinions in Cell Biology 5 613-620 